\title{
Esters of quinoxaline-7-carboxylate-1,4-di- $N$-oxide as Trichomonas vaginalis triosephosphate isomerase inhibitors
}

\author{
ISIDRO PALOS \\ ROSA MOO-PUC ${ }^{2}$ \\ JOSÉ LUIS VIQUE-SÁNCHEZ 3,6 \\ CLAUDIA G. BENÍTEZ-CARDOZA ${ }^{3}$ \\ ANTONIO MONGE 4 \\ JUAN CARLOS VILLALOBOS-ROCHA \\ ALMA D. PAZ-GONZALEZ \\ GILDARDO RIVERA ${ }^{5,7}$ \\ ${ }^{1}$ Unidad Académica Multidisciplinaria \\ Reynosa-Rodhe, Universidad Autónoma de \\ Tamaulipas, 88779 Reynosa, México \\ ${ }^{2}$ Unidad de Investigación Médica Yucatán, Unidad \\ Médica de Alta Especialidad, Centro Médico Ignacio \\ García Téllez, Instituto Mexicano del Seguro Social \\ Col. Industrial, 97150 Mérida, México
}

${ }^{3}$ Laboratorio de Investigación Bioquímica Escuela Nacional de Medicina y Homeopatía Instituto Politécnico Nacional, Guillermo Massieu Helguera No. 239, La Escalera Ticoman 07320 Ciudad de México, México

${ }^{4}$ Neglected Diseases Section, Drug R \& D Unit Center for Applied Pharmacobiology Research University of Navarra, C/Irunlarrea, 31008 Pamplona, Spain

${ }^{5}$ Laboratorio de Biotecnología Farmacéutica, Centro de Biotecnología Genómica, Instituto Politécnico Nacional, 88710 Reynosa, México

${ }^{6}$ Facultad de Medicina, Universidad Autónoma de Baja California, Mexicali, México

Accepted October 9, 2020

Published online November 10, 2020
Trichomoniasis is a public health problem worldwide. Trichomoniasis treatment consists of the use of nitroimidazole derivatives; however, therapeutic ineffectiveness occurs in 5 to $20 \%$ of the cases. Therefore, it is essential to propose new pharmacological agents against this disease. In this work, esters of quinoxaline-7-carboxylate-1,4-di- $N$-oxide (EQX-NO) were evaluated in in vitro assays as novel trichomonicidal agents. Additionally, an in vitro enzyme assay and molecular docking analysis against triosephosphate isomerase of Trichomonas vaginalis to confirm their mechanism of action were performed. Ethyl (compound 12) and n-propyl (compound 37) esters of quinoxaline-7-carboxylate-1,4-di- $N$-oxide derivatives showed trichomonicidal activity comparable to nitazoxanide, whereas five methyl (compounds 5, 15, 19, 20 and 22), four isopropyl (compounds 28, 29, 30 and 34), three ethyl (compounds 4, 13 and 23) and one npropyl (compound 35) ester derivatives displayed activity comparable to albendazole. Compounds 6 and 20 decreased $100 \%$ of the enzyme activity of recombinant protein triosephosphate isomerase.

Keywords: quinoxaline 1,4-di-N-oxide, trichomoniasis, triosephosphate isomerase inhibitor

Trichomonas vaginalis (T. vaginalis) is a protozoon that poses an important public health challenge (1-3). It colonizes the human urogenital tract by adhering to the epithelium. T. vaginalis infection is often asymptomatic but can produce inflammation of the urinary

*Correspondence; e-mail address: gildardors@hotmail.com 
tract. Trichomoniasis is a non-viral sexually diffused infection that affects approximately 276 million people per year $(4,5)$.

The drug of choice for trichomoniasis is metronidazole, a 5-nitroimidazole derivative (5); however, it causes severe adverse effects since it is carcinogenic and teratogenic (6). These effects have been associated with prolonged treatment or high doses $(7,8)$. Additionally, the expansion of resistant T. vaginalis strains makes new antitrichomoniasis agents necessary.

Quinoxaline (QX) is a privileged structure for the design and development of new antibacterial (9), anticancer (10), and antiparasitic (11) agents with some derivatives in the clinical phase studies $(12,13)$. Interestingly, quinoxaline 1,4 -di- $N$-oxide $(\mathrm{QX}-\mathrm{NO})$ derivatives show biological effects against diverse protozoa (14-17); however, QX-NO has not been evaluated against $T$. vaginalis. Therefore, in this work, the esters of quinoxaline-7-carboxylate-1,4-di-N-oxide (EQX-NO) derivatives were evaluated as novel antitrichomoniasis agents. Furthermore, in silico and in vitro analyses were done against T. vaginalis triosephosphate isomerase to elucidate their mechanism of action.

\section{EXPERIMENTAL}

Syntheses

All EQX-NO derivatives were synthesized using the method reported by Gomez-Caro et al. (18). All molecules were identified by IR spectroscopy, ${ }^{1} \mathrm{H}$ NMR and elemental analysis of C, H and N (see Table SI, Supplementary material), as in previous research $(14,15,17$, 18).

\section{In vitro evaluation against $\mathrm{T}$. vaginalis}

T. vaginalis strain GT3 (a wild strain isolated in Centro Medico Nacional Siglo XXI, Instituto Mexicano del Seguro Social, Mexico) was used. EQX-NO derivatives, albendazole (ABZ), metronidazole (MTZ), and nitazoxanide (NTZ) were tested against $T$. vaginalis strain GT3 in vitro using the previously reported method (19). Dimethylsulfoxide (DMSO) was used as a negative control. The half-maximal inhibitory concentration $\left(I C_{50}\right)$ was calculated using the Probit analysis.

\section{In silico procedures}

Protein structure. - The atomic coordinates of triosephosphate isomerase (TIM) from $T$. vaginalis (TvTIM) from the Protein Data Bank (PDB, code 3QSR) were used with Molecular Operating Environment (MOE) software and CHARMM27 force field $(20,21)$. This structure was used to predict binding free energy $\left(\Delta G_{\text {bind }}\right)$ values between TvTIM and EQX-NO derivatives. Site Finder implemented in the MOE was used to determine potential binding sites $(22,23)$.

Molecular docking. - For molecular docking, the receptor (TIM, 3QSR) was kept inflexible, while examining twenty conformations for each of the ligands. All crystallographic water molecules were deleted from the initial structures. Molecular docking was done 
with the MOE software using the previously reported procedure $(24,25)$. The molecules were analyzed on twenty-three potential binding sites from TvTIM (Table SII, Supplementary material) to propose a potential interaction site that explains the effect on enzyme activity.

To describe the interaction of the ligands, the binding affinity of each complex was estimated with the generalized Born/volume integral ratio (GB/VI) using parameters in MOE (26). The generalized Born or non-bonded interaction energies comprised van der Waals, Coulomb electrostatic interactions, and implied solvent interaction energies.

\section{In vitro assays against TvTIM}

Stock solutions of EQX-NO derivatives were prepared in DMSO (1 \%) and further diluted with media to obtain 50 and $100 \mu \mathrm{g} \mu \mathrm{L}^{-1}$ of each compound tested. Recombinant TvTIM was obtained as previously described $(27,28)$. The conversion of glyceraldehyde 3-phosphate (GAP) into dihydroxyacetone phosphate was followed to determine enzyme activity following a previous procedure (29-35).

\section{RESULTS AND DISCUSSION}

Methyl (m-), ethyl (e-), isopropyl ( $i-)$, and $n$-propyl (np-) EQX-NO derivatives were obtained (see Supplementary material) (18) and evaluated in vitro against $T$. vaginalis. Table I shows their trichomonicidal activity.

\section{Trichomonicidal activity}

Among m- and e-EQX-NO derivatives, twelve compounds had an $I C_{50}$ between 1 and $10 \mu \mathrm{mol} \mathrm{L}-1$, and seven compounds had a higher $I C_{50}$ value than ABZ (Table I). However, none of the m- and e-EQX-NO derivatives had trichomonicidal activity greater than MTZ and NTZ. Only compound 12, an ethyl methyl-3-methyl-quinoxaline-2,7-dicarboxylate-1,4di- $N$ - oxide, had $I C_{50}$ of $0.97 \mu \mathrm{mol} \mathrm{L}^{-1}$, a value comparable or equal to both reference drugs (MTZ and NTZ). In general, the structure-activity relationship (SAR) analysis showed that aliphatic, ethoxy and methoxy groups at R1-position on the QX ring enhance biological effects. Additionally, the steric effect at R1-position is a key factor in the activity. A trifluoromethyl and a methyl group at R2- and R3-position, resp., also showed trichomonicidal activity.

None of the five $n$ p-EQX-NO derivatives showed trichomonicidal activity, but two compounds had $I C_{50}$ values from 5.0 to $6.2 \mu \mathrm{mol} \mathrm{L}{ }^{-1}$, whereas two more had an $I C_{50}$ value $<3 \mu \mathrm{mol} \mathrm{L}^{-1}$ (Table I). Compounds 35 (n-propyl methyl-3-methylquinoxaline-2,7-dicarboxylate-1,4-di- $N$-oxide) and 37 (n-propyl ethyl-3-methylquinoxaline-2,7-dicarboxylate-1,4-di$-N$-oxide) showed better trichomonicidal activity than ABZ. Both compounds have an ester and methyl group at R1- and R2-position, resp., on the QX-NO ring. Additionally, compound 37 showed activity comparable to NTZ.

From $i$-EQX-NO derivatives, nine compounds showed no trichomonicidal activity $\left(I C_{50}>10 \mu \mathrm{mol} \mathrm{L}-1\right)$ and eight compounds had an $I C_{50}$ value from 2.9 to $6.4 \mu \mathrm{mol} \mathrm{L}-1$. 
Table I. The half-maximal inhibitory concentration $\left(\mathrm{IC}_{50}\right)$ of $\mathrm{EQX}-\mathrm{NO}$ derivatives against $\mathrm{T}$. vaginalis<smiles>[R]OC(=O)c1ccc2c(c1)[n+]([O-])c(C([R])=O)c([R1])[n+]2[O-]</smiles>

\begin{tabular}{|c|c|c|c|c|}
\hline Compd. & $\mathrm{R}_{1}$ & $\mathrm{R}_{2}$ & $\mathrm{R}_{3}$ & $I C_{50}\left(\mu \mathrm{mol} \mathrm{L}^{-1}\right)^{\mathrm{a}}$ \\
\hline 1 & $\mathrm{C}_{6} \mathrm{H}_{5}$ & $\mathrm{CH}_{3}$ & $\mathrm{CH}_{3}$ & $>10$ \\
\hline 2 & $\mathrm{C}_{6} \mathrm{H}_{5}$ & $\mathrm{CH}_{3}$ & $\mathrm{CH}_{3} \mathrm{CH}_{2}$ & $>10$ \\
\hline 3 & $\mathrm{CH}_{3}$ & $\mathrm{CF}_{3}$ & $\mathrm{CH}_{3} \mathrm{CH}_{2}$ & $>10$ \\
\hline 4 & $\mathrm{C}_{10} \mathrm{H}_{7}$ & $\mathrm{CF}_{3}$ & $\mathrm{CH}_{3} \mathrm{CH}_{2}$ & $3.52 \pm 0.07$ \\
\hline 5 & $\mathrm{C}_{4} \mathrm{H}_{3} \mathrm{~S}$ & $\mathrm{CF}_{3}$ & $\mathrm{CH}_{3}$ & $2.63 \pm 0.01$ \\
\hline 6 & $\mathrm{C}_{4} \mathrm{H}_{3} \mathrm{~S}$ & $\mathrm{CF}_{3}$ & $\mathrm{CH}_{3} \mathrm{CH}_{2}$ & $>10$ \\
\hline 7 & $\mathrm{NHC}_{6} \mathrm{H}_{5}$ & $\mathrm{CH}_{3}$ & $\mathrm{CH}_{3}$ & $>10$ \\
\hline 8 & $\mathrm{OCH}_{2} \mathrm{CH}_{3}$ & $\mathrm{CH}_{3}$ & $\mathrm{CH}_{3}$ & $>10$ \\
\hline 9 & $\mathrm{OCH}_{2} \mathrm{CH}_{3}$ & $\mathrm{CH}_{3}$ & $\mathrm{CH}_{3} \mathrm{CH}_{2}$ & $5.93 \pm 0.23$ \\
\hline 10 & $\mathrm{C}_{6} \mathrm{H}_{5}$ & $\mathrm{CF}_{3}$ & $\mathrm{CH}_{3}$ & $5.32 \pm 0.12$ \\
\hline 11 & $\mathrm{OCH}_{3}$ & $\mathrm{CH}_{3}$ & $\mathrm{CH}_{3}$ & $6.19 \pm 0.32$ \\
\hline 12 & $\mathrm{OCH}_{3}$ & $\mathrm{CH}_{3}$ & $\mathrm{CH}_{3} \mathrm{CH}_{2}$ & $0.97 \pm 0.04$ \\
\hline 13 & $\mathrm{CH}_{2} \mathrm{CH}_{3}$ & $\mathrm{CF}_{3}$ & $\mathrm{CH}_{3} \mathrm{CH}_{2}$ & $2.51 \pm 0.09$ \\
\hline 14 & $\mathrm{C}_{6} \mathrm{H}_{5} \mathrm{Cl}$ & $\mathrm{CF}_{3}$ & $\mathrm{CH}_{3} \mathrm{CH}_{2}$ & $>10$ \\
\hline 15 & $\mathrm{C}_{10} \mathrm{H}_{7}$ & $\mathrm{CF}_{3}$ & $\mathrm{CH}_{3}$ & $4.29 \pm 0.12$ \\
\hline 16 & $\mathrm{C}_{4} \mathrm{H}_{3} \mathrm{O}$ & $\mathrm{CF}_{3}$ & $\mathrm{CH}_{3}$ & $>10$ \\
\hline 17 & $\mathrm{CH}_{3}$ & $\mathrm{CH}_{3}$ & $\mathrm{CH}_{3}$ & $>10$ \\
\hline 18 & $\mathrm{OCH}_{2} \mathrm{CH}_{3}$ & $\mathrm{CH}_{2} \mathrm{COOCH}_{2} \mathrm{CH}_{3}$ & $\mathrm{CH}_{3}$ & $>10$ \\
\hline 19 & $\mathrm{CH}_{2} \mathrm{CH}_{3}$ & $\mathrm{CF}_{3}$ & $\mathrm{CH}_{3}$ & $3.25 \pm 0.05$ \\
\hline 20 & $\mathrm{CH}\left(\mathrm{CH}_{3}\right)_{2}$ & $\mathrm{CF}_{3}$ & $\mathrm{CH}_{3}$ & $4.01 \pm 0.11$ \\
\hline 21 & $\mathrm{OCH}_{2} \mathrm{C}_{6} \mathrm{H}_{5}$ & $\mathrm{CH}_{3}$ & $\mathrm{CH}_{3}$ & $>10$ \\
\hline 22 & $\mathrm{OCH}_{2} \mathrm{CH}_{3}$ & $\mathrm{C}_{6} \mathrm{H}_{5}$ & $\mathrm{CH}_{3}$ & $2.55 \pm 0.03$ \\
\hline 23 & $\mathrm{OCH}_{2} \mathrm{CH}_{3}$ & $\mathrm{C}_{6} \mathrm{H}_{5}$ & $\mathrm{CH}_{3} \mathrm{CH}_{2}$ & $2.92 \pm 0.01$ \\
\hline 24 & $\mathrm{NH}_{2}$ & $\mathrm{CH}_{3}$ & $\mathrm{CH}_{3} \mathrm{CH}_{2}$ & $6.00 \pm 0.04$ \\
\hline 25 & $\mathrm{OCH}_{3}$ & $\mathrm{CH}_{3}$ & $\left(\mathrm{CH}_{3}\right)_{2} \mathrm{CH}$ & $>10$ \\
\hline 26 & $\mathrm{OCH}_{2} \mathrm{CH}_{3}$ & $\mathrm{CH}_{3}$ & $\left(\mathrm{CH}_{3}\right)_{2} \mathrm{CH}$ & $>10$ \\
\hline 27 & $\mathrm{OC}\left(\mathrm{CH}_{3}\right)_{3}$ & $\mathrm{CH}_{3}$ & $\left(\mathrm{CH}_{3}\right)_{2} \mathrm{CH}$ & $>10$ \\
\hline 28 & $\mathrm{OCH}_{2} \mathrm{CH}_{3}$ & $\mathrm{CH}_{2} \mathrm{COOCH}_{2} \mathrm{CH}_{3}$ & $\left(\mathrm{CH}_{3}\right)_{2} \mathrm{CH}$ & $3.50 \pm 0.12$ \\
\hline 29 & $\mathrm{C}_{4} \mathrm{H}_{3} \mathrm{~S}$ & $\mathrm{CF}_{3}$ & $\left(\mathrm{CH}_{3}\right)_{2} \mathrm{CH}$ & $3.68 \pm 0.04$ \\
\hline
\end{tabular}




\begin{tabular}{|c|c|c|c|c|}
\hline Compd. & $\mathrm{R}_{1}$ & $\mathrm{R}_{2}$ & $\mathrm{R}_{3}$ & $I C_{50}\left(\mu \mathrm{mol} \mathrm{L}^{-1}\right)^{\mathrm{a}}$ \\
\hline 30 & $\mathrm{CH}_{3}$ & $\mathrm{CF}_{3}$ & $\left(\mathrm{CH}_{3}\right)_{2} \mathrm{CH}$ & $3.77 \pm 0.02$ \\
\hline 31 & $\mathrm{C}_{6} \mathrm{H}_{5}$ & $\mathrm{CF}_{3}$ & $\left(\mathrm{CH}_{3}\right)_{2} \mathrm{CH}$ & $5.25 \pm 0.01$ \\
\hline 32 & $\mathrm{C}_{10} \mathrm{H}_{7}$ & $\mathrm{CF}_{3}$ & $\left(\mathrm{CH}_{3}\right)_{2} \mathrm{CH}$ & $5.41 \pm 0.01$ \\
\hline 33 & $\mathrm{C}_{4} \mathrm{H}_{3} \mathrm{O}$ & $\mathrm{CF}_{3}$ & $\left(\mathrm{CH}_{3}\right)_{2} \mathrm{CH}$ & $>10$ \\
\hline 34 & $\mathrm{CH}\left(\mathrm{CH}_{3}\right)_{2}$ & $\mathrm{CF}_{3}$ & $\left(\mathrm{CH}_{3}\right)_{2} \mathrm{CH}$ & $3.21 \pm 0.07$ \\
\hline 35 & $\mathrm{OCH}_{3}$ & $\mathrm{CH}_{3}$ & $\mathrm{CH}_{3} \mathrm{CH}_{2} \mathrm{CH}_{2}$ & $2.56 \pm 0.11$ \\
\hline 36 & $\mathrm{C}_{4} \mathrm{H}_{3} \mathrm{~S}$ & $\mathrm{CF}_{3}$ & $\mathrm{CH}_{3} \mathrm{CH}_{2} \mathrm{CH}_{2}$ & $5.04 \pm 0.12$ \\
\hline 37 & $\mathrm{OCH}_{2} \mathrm{CH}_{3}$ & $\mathrm{CH}_{3}$ & $\mathrm{CH}_{3} \mathrm{CH}_{2} \mathrm{CH}_{2}$ & $1.46 \pm 0.01$ \\
\hline 38 & $\mathrm{C}\left(\mathrm{CH}_{3}\right)_{3}$ & $\mathrm{CH}_{3}$ & $\mathrm{CH}_{3} \mathrm{CH}_{2} \mathrm{CH}_{2}$ & $>10$ \\
\hline 39 & $\mathrm{NHC}_{6} \mathrm{H}_{5}$ & $\mathrm{CH}_{3}$ & $\left(\mathrm{CH}_{3}\right)_{2} \mathrm{CH}$ & $>10$ \\
\hline 40 & $\mathrm{C}_{6} \mathrm{H}_{5}$ & $\mathrm{CH}_{3}$ & $\left(\mathrm{CH}_{3}\right)_{2} \mathrm{CH}$ & $6.46 \pm 0.08$ \\
\hline 41 & $\mathrm{C}\left(\mathrm{CH}_{3}\right)_{3}$ & $\mathrm{C}\left(\mathrm{CH}_{3}\right)_{3}$ & $\left(\mathrm{CH}_{3}\right)_{2} \mathrm{CH}$ & $>10$ \\
\hline 42 & $\mathrm{NH}_{2}$ & $\mathrm{CH}_{3}$ & $\left(\mathrm{CH}_{3}\right)_{2} \mathrm{CH}$ & $>10$ \\
\hline 43 & $\mathrm{C}_{4} \mathrm{H}_{3} \mathrm{O}$ & $\mathrm{CF}_{3}$ & $\mathrm{CH}_{3} \mathrm{CH}_{2} \mathrm{CH}_{2}$ & $>10$ \\
\hline 44 & $\mathrm{OCH}_{2} \mathrm{CH}_{3}$ & $\mathrm{CF}_{3}$ & $\left(\mathrm{CH}_{3}\right)_{2} \mathrm{CH}$ & $2.98 \pm 0.07$ \\
\hline 45 & $\mathrm{C}\left(\mathrm{CH}_{3}\right)_{3}$ & $\mathrm{CF}_{2} \mathrm{CF}_{2} \mathrm{CF}_{3}$ & $\left(\mathrm{CH}_{3}\right)_{2} \mathrm{CH}$ & $>10$ \\
\hline 46 & $\mathrm{CF}_{2} \mathrm{CF}_{3}$ & $\mathrm{CF}_{3}$ & $\left(\mathrm{CH}_{3}\right)_{2} \mathrm{CH}$ & $>10$ \\
\hline 47 & $\mathrm{OCH}_{2} \mathrm{CH}_{3}$ & $\mathrm{CF}_{3}$ & $\mathrm{CH}_{3} \mathrm{CH}_{2} \mathrm{CH}_{2}$ & $>10$ \\
\hline 48 & $\mathrm{NHC}_{6} \mathrm{H}_{5}$ & $\mathrm{CH}_{3}$ & $\mathrm{CH}_{3} \mathrm{CH}_{2} \mathrm{CH}_{2}$ & $>10$ \\
\hline 49 & $\mathrm{CH}_{3}$ & $\mathrm{CH}_{3}$ & $\mathrm{CH}_{3} \mathrm{CH}_{2} \mathrm{CH}_{2}$ & $6.24 \pm 0 . .41$ \\
\hline 50 & $\mathrm{NH}_{2}$ & $\mathrm{CH}_{3}$ & $\mathrm{CH}_{3} \mathrm{CH}_{2} \mathrm{CH}_{2}$ & $>10$ \\
\hline $51^{\mathrm{b}}$ & $\mathrm{OCH}_{3}$ & $\mathrm{CH}_{3}$ & $\mathrm{CH}_{2} \mathrm{CH}_{3}$ & $>10$ \\
\hline $52^{b}$ & $\mathrm{C}_{6} \mathrm{H}_{5}$ & $\mathrm{CF}_{3}$ & $\mathrm{CH}_{3}$ & $4.94 \pm 0.12$ \\
\hline $53^{b}$ & $\mathrm{CH}\left(\mathrm{CH}_{3}\right)_{2}$ & $\mathrm{CF}_{3}$ & $\mathrm{CH}_{3}$ & $>10$ \\
\hline $\mathrm{ABZ}$ & & & & $4.08 \pm 0.21$ \\
\hline MTZ & & & & $0.69 \pm 0.01$ \\
\hline NTZ & & & & $1.00 \pm 0.05$ \\
\hline
\end{tabular}

ABZ - albendazole, MTZ - metronidazole, NTZ - nitazoxanide

${ }^{a}$ Mean \pm SD of triplicate determinations.

${ }^{\mathrm{b}}$ Quinoxaline derivatives without $\mathrm{N}$-oxide groups.

Although five compounds had lower $I C_{50}$ values $\left(<4.0 \mu \mathrm{mol} \mathrm{L}^{-1}\right)$ than $\mathrm{ABZ}$, none showed better trichomonicidal activity than MTZ and NTZ (Table I).

Finally, the $N$-oxide groups on the QX ring from some EQX-NO derivatives were eliminated (by reduction with sodium dithionite) to determine their effect on trichomonicidal activity (Table I). EQX-NO derivatives 10, 12, and 20 with trichomonicidal activity below 
$6.0 \mu \mathrm{mol} \mathrm{L} \mathrm{L}^{-1}$ were selected to obtain QX derivatives 51, 52, and 53 (see Supplementary material), resp. These new compounds showed no or low biological activity, except compound 52 (methyl 2-benzoyl-3-trifluoromethyl quinoxaline-7-carboxylate), which had an antiprotozoal effect against T. vaginalis. These results show that the $\mathrm{N}$-oxide groups are the key factor for the trichomonicidal activity and suggest that compound $\mathbf{5 2}$ has a different mechanism of action than EQX-NO derivatives. Still, the mechanism of action of QX-NO is currently not clear, as reported by Cheng et al. (12).

\section{Enzymatic activity of ToTIM}

Previously, Guzman et al. (32), reported that QX-NO derivatives affect the enzymatic activity of Trypanosoma cruzi TIM. Therefore, in this work, TvTIM was considered a potential drug target. All EQX-NO derivatives were evaluated in enzyme activity assays, but only four compounds $(\mathbf{6}, \mathbf{1 0}, 20$ and 42) decreased TvTIM activity to some degree. The effect of compounds 6 (ethyl 2-(thiophene-2-carbonyl)-3-trifluoromethylquinoxaline-7-carboxylate-1,4-di- $\mathrm{N}$-oxide), 10 (methyl 2-benzoyl-3-trifluoromethylquinoxaline-7-carboxyl-

Table II. Effect of EQX-NO derivatives against the enzymatic activity of TvTIM, predictive binding free energy value and main residues in the interaction on the active site

\begin{tabular}{ccccc}
\hline Compd. & \multicolumn{2}{c}{ TvTIM enzymatic activity $(\%)^{\mathrm{a}}$} & $\begin{array}{c}\Delta G_{\text {bind }} \\
\left(\mathrm{kcal} \mathrm{mol}^{-1}\right)\end{array}$ & $\begin{array}{c}\text { Main amino acids in the } \\
\text { interactions }\end{array}$ \\
\hline $\mathbf{6}$ & $50 \mathrm{~g} \mathrm{~L} \mathrm{~L}^{-1}$ & 0 & -0.6 & Glu166 \\
$\mathbf{1 0}$ & $69.6 \pm 4.1$ & $38.3 \pm 3.2$ & -7.3 & Lys11, Gly172, and Ser212 \\
$\mathbf{2 0}$ & $49.6 \pm 2.5$ & 0 & -3.2 & Lys11, Ile171, and Gly172 \\
$\mathbf{4 2}$ & $51.6 \pm 4.1$ & $30.3 \pm 4.0$ & -4.7 & Lys11, Ala170, Ile171, and Gly172 \\
\hline
\end{tabular}

$\Delta G_{\text {bind }}$-binding free energy

${ }^{\text {a }}$ Mean \pm SD of triplicate determinations.

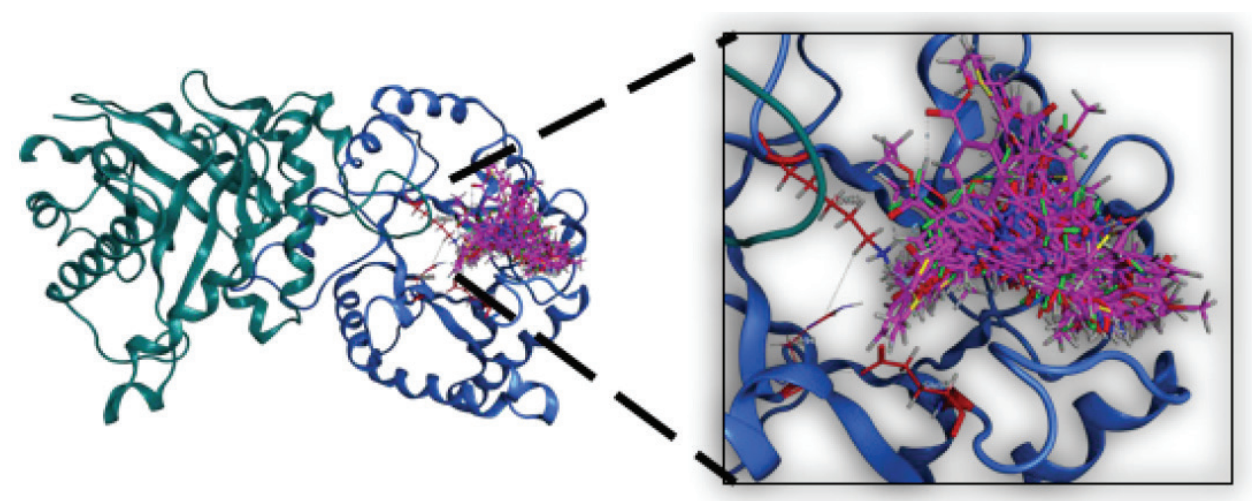

Fig. 1. The main interaction site on TvTIM for the compounds 6, 10, 20, and 42. Residues Lys11, His94, and Glu166 (red color) and seven conformers of each compound (pink color). 
ate-1,4-di- $N$-oxide), 20 (ethyl methyl-3-methyl-quinoxaline-2,7-dicarboxylate-1,4-di- $\mathrm{N}$-oxide), and 42 (isopropyl 2-amide-3-methyl-quinoxaline-7-carboxylate-1,4-di- $\mathrm{N}$-oxide) on the activity of TvTIM was determined in two concentrations (50 and $100 \mu \mathrm{g} \mu \mathrm{L}^{-1}$ ). The results are displayed in Table II. Three of the abovementioned EQX-NO derivatives (6, 20, and 42) showed similar enzyme activity inhibition (50\% approx.) at $50 \mu \mathrm{g} \mu \mathrm{L}^{-1}$, and only compounds 6 and 20 showed $100 \%$ enzyme inhibition at $100 \mu \mathrm{g} \mu \mathrm{L}^{-1}$. These compounds caused a decrease in the function of TVTIM at higher concentrations than the other reported compounds (34-36). Nevertheless, this is the first report that shows that EQX-NO derivatives have an inhibitory effect against TvTIM. Previously, Cheng et al. (12) mentioned two sulfoxide QX-NO derivatives with a better trichonomicidal activity than metronidazole, however, the mechanism of action was not explored. Therefore, QX-NO derivatives are a new option to develop new TvTIM inhibitors.

a)

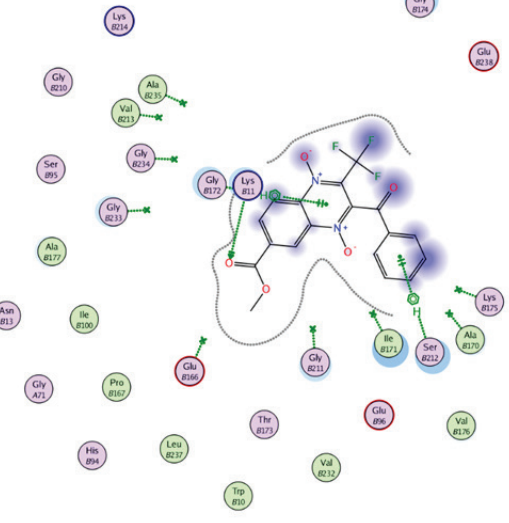

c)

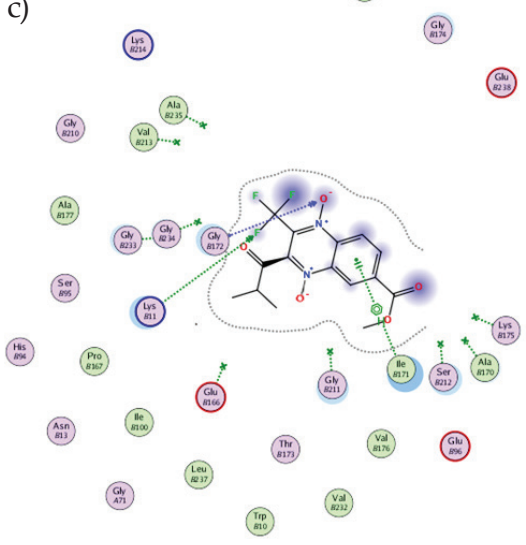

b)

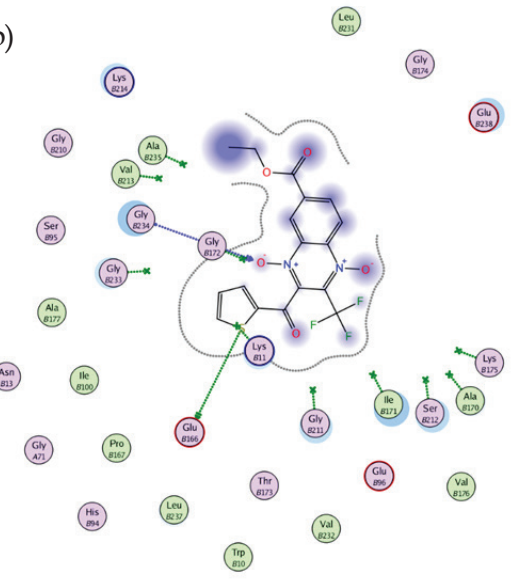

(20)

d)

(圈)

(aik

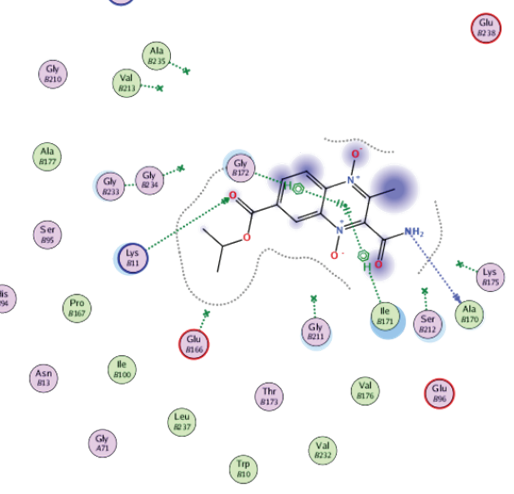

Fig. 2. Interaction site between EQX-NO derivatives and the potential active site of TvTIM. a) compound $\mathbf{6}, \mathrm{b}$ ) compound $\mathbf{1 0 ,}$ ) compound 20, and d) compound $\mathbf{4 2}$. 


\section{Molecular docking analysis on ToTIM}

Molecular docking analysis was done to describe the molecular interaction of the EQXNO derivatives on 23 potential TvTIM binding sites (Table SII, Supplementary material).

The compounds that affected the enzymatic activity $(\mathbf{6}, \mathbf{1 0}, \mathbf{2 0}$, and 42$)$ showed the best interactions with Lys11, His94, and Glu166, among other residues, near the active site (Fig. 1). The best interactions for the four compounds were determined in the region of residues near Lys11, His94, Glu166, Ala170, Ile171, Gly172, and Ser212 (Fig. 2). Additionally, predictive $\Delta G_{\text {bind }}$ was determined (Table II). Compound 10 showed the lowest $\Delta G_{\text {bind }}$ value $\left(-7.3 \mathrm{kcal} \mathrm{mol}^{-1}\right)$; however, this compound showed the lowest enzyme inhibition (31\%) at 50 $\mu \mathrm{g} \mu \mathrm{L}^{-1}$. The other three compounds $(\mathbf{6}, 20$, and 42$)$ showed very different $\Delta G_{\text {bind }}$ values but comparable enzyme inhibition. These results suggest no correlation between in vitro and in silico analysis. Therefore, compounds $\mathbf{6}, \mathbf{1 0}, \mathbf{2 0}$, and 42 could be interacting on another binding site of TvTIM, causing an effect on the enzyme activity assays. The molecular docking results from the other QX-NO derivatives showed a probable interaction in the interface and core of TvTIM, which can be related to not affecting the enzymatic activity of TvTIM.

\section{CONCLUSIONS}

Four different types of EQX-NO derivatives were tested as novel agents against T. vaginalis. e-EQX-NO derivatives showed a better trichomonicidal effect than BZN. In particular, compound 12 (ethyl methyl-3-methyl-quinoxaline-2,7-dicarboxylate-1,4-di- $N$-oxide) in vitro assays show a better trichomonicidal activity than ABZ and NTZ. Additionally, four compounds 6 (ethyl 2-(thiophene-2-carbonyl)-3-trifluoromethylquinoxaline-7-carboxylate-1,4-di- $\mathrm{N}$-oxide), 10 (methyl 2-benzoyl-3-trifluoromethylquinoxaline-7-carboxylate-1,4-di- $\mathrm{N}$-oxide), 20 ( ethyl methyl-3-methyl-quinoxaline-2,7-dicarboxylate-1,4-di- $N$-oxide), and 42 (isopropyl 2-amide-3-methyl-quinoxaline-7-carboxylate-1,4-di- $N$-oxide) reduced the enzymatic activity of recombinant protein TVTIM. SAR analysis showed that methyl and ethyl groups at R3position and $\mathrm{N}$-oxide groups on the QX ring are the key moieties for the biological activity. Molecular docking analysis revealed that the main interaction site of these compounds is near to the active site where they interact with Lys11, Glu166, Ala170, Ile171, and Gly172. Therefore, these types of compounds could help in the development of new and more effective TvTIM inhibitors to combat the T. vaginalis resistance.

Acknowledgments. - Supplementary materials available upon request.

\section{REFERENCES}

1. D. Leitsch, Drug resistance in the microaerophilic parasite Giardia lamblia, Curr. Trop. Med. Rep. 2 (2015) 128-135; https://doi.org/10.1007/s40475-015-0051-1

2. B. R. Ansell, M. J. McConville, S. Y. Ma'ayeh, M. J. Dagley, R. B. Gasser, S. G. Svärd and A. R. Jex, Drug resistance in Giardia duodenalis, Biotechnol. Adv. 33 (2015) 888-901; https://doi.org/10.1016/j. biotechadv.2015.04.009

3. C. B. Menezes, A. P. Frasson and T. Tasca, Trichomoniasis - are we giving the deserved attention to the most common non-viral sexually transmitted disease worldwide?, Microb. Cell 3 (2016) 404419; https://doi.org/10.15698/mic2016.09.526 
I. Palos et al.: Esters of quinoxaline-7-carboxylate-1,4-di-N-oxide as Trichomonas vaginalis triosephosphate isomerase inhibitors, Acta Pharm. 71 (2021) 485-495.

4. D. Leitsch, Recent advances in the Trichomonas vaginalis field, F1000Res. 5 (2016) Article ID 162 (7 pages); https://doi.org/10.12688/f1000research.7594.1

5. P. Kissinger, Trichomonas vaginalis: a review of epidemiologic, clinical and treatment issues, BMC Infect. Dis. 15 (2015) Article ID 307 (8 pages); https://doi.org/10.1186/s12879-015-1055-0

6. P. Upcroft and J. A. Upcroft, Drug targets and mechanisms of resistance in the anaerobic protozoa, Clin. Microbiol. Rev. 14 (2001) 150-164; https://doi.org/10.1128/CMR.14.1.150-164.2001

7. P. A. Cano, A. Islas-Jácome, J. González-Marrero, L. Yépez-Mulia, F. Calzada and R. Gámez-Montaño, Synthesis of 3-tetrazolylmethyl-4H-chromen-4-ones via Ugi-azide and biological evaluation against Entamoeba histolytica, Giardia lamblia and Trichomona vaginalis, Bioorg. Med. Chem. 22 (2014) 1370-1376; https://doi.org/10.1016/j.bmc.2013.12.069

8. S. Chaturvedi, M. Y. Malik, M. Rashid, S. Singh, V. Tiwari, P. Gupta, S. Shukla, S. Singh and M. Wahajuddin, Mechanistic exploration of quercetin against metronidazole induced neurotoxicity in rats: possible role of nitric oxide isoforms and inflammatory cytokines, Neurotoxicology 79 (2020) 1-10; https://doi.org/10.1016/j.neuro.2020.03.002

9. J. Jampilek, Recent advances in design of potential quinoxaline anti-infectives, Curr. Med. Chem. 21 (2014) 4347-4373; https://doi.org/10.2174/0929867321666141011194825

10. I. Balderas-Renteria, P. Gonzalez-Barranco, A. Garcia, B. K. Banik and G. Rivera, Anticancer drug design using scaffolds of $\beta$-lactams, sulfonamides, quinoline, quinoxaline and natural products. Drugs advances in clinical trials, Curr. Med. Chem. 19 (2012) 4377-4398; https://doi. org/10.2174/092986712803251593

11. N. B. Patel, J. N. Patel, A. C. Purohit, V. M. Patel, D. P. Rajani, R. Moo-Puc, J. C. Lopez-Cedillo, B. Nogueda-Torres and G. Rivera, In vitro and in vivo assessment of newer quinoxaline-oxadiazole hybrids as antimicrobial and antiprotozoal agents, Int. J. Antimicrob. Agents 50 (2017) 413-418; https://doi.org/10.1016/j.ijantimicag.2017.04.016

12. G. Cheng, W. Sa, C. Cao, L. Guo, H. Hao, Z. Liu, X. Wang and Z. Yuan, Quinoxaline 1,4-di-N-oxides: Biological activities and mechanisms of actions, Front. Pharmacol. 7 (2016) Article ID 64 (21 pages); https://doi.org/10.3389/fphar.2016.00064

13. R. El Aissi, J. Liu, S. Besse, D. Canitrot, O. Chavignon, J. M. Chezal, E. Miot-Noirault and E. Moreau, Synthesis and biological evaluation of new quinoxaline derivatives of ICF01012 as melanoma-targeting probes, ACS Med. Chem. Lett. 5 (2014) 468-473; https://doi.org/10.1021/ml400468x

14. J. C. Villalobos-Rocha, L. Sánchez-Torres, B. Nogueda-Torres, A. Segura-Cabrera, C. A. GarcíaPérez, V. Bocanegra-García, I. Palos, A. Monge and G. Rivera, Anti-Trypanosoma cruzi and antileishmanial activity by quinoxaline-7-carboxylate 1,4-di-N-oxide derivatives, Parasitol. Res. 113 (2014) 2027-2035; https://doi.org/10.1007/s00436-014-3850-8

15. K. F. Chacón-Vargas, S. Andrade-Ochoa, B. Nogueda-Torres, D. C. Juárez-Ramírez, E. E. LaraRamírez, R. Mondragón-Flores, A. Monge and G. Rivera, L. E. Sánchez-Torres, Isopropyl quinoxaline-7-carboxylate 1,4-di-N-oxide derivatives induce regulated necrosis-like cell death on Leishmania (Leishmania) mexicana, Parasitol. Res. 117 (2018) 45-58; https://doi.org/10.1007/ s00436-017-5635-3

16. M. Quiliano, A. Pabón, G. Ramirez-Calderon, C. Barea, E. Deharo, S. Galiano and I. Aldana, New hydrazine and hydrazide quinoxaline 1,4-di-N-oxide derivatives: In silico ADMET, antiplasmodial and antileishmanial activity. Bioorg. Med. Chem. Lett. 27 (2017) 1820-1825; https://doi. org/10.1016/j.bmcl.2017.02.049

17. B. E. Duque-Montaño, L. C. Gómez-Caro, M. Sanchez-Sanchez, A. Monge, E. Hernández-Baltazar, G. Rivera and O. Torres-Angeles, Synthesis and in vitro evaluation of new ethyl and methyl quinoxaline-7-carboxylate 1,4-di-N-oxide against Entamoeba histolytica, Bioorg. Med. Chem. 21 (2013) 4550-4558; https://doi.org/10.1016/j.bmc.2013.05.036 
18. L. C. Gómez-Caro, M. Sánchez-Sánchez, V. Bocanegra-García, A. Monge and G. Rivera, Synthesis of quinoxaline 1,4-di- $\mathrm{N}$-oxide derivatives on solid support using room temperature and microwave-assisted solvent-free procedures, Quim. Nova 34 (2011) 1147-1151; https://doi.org/10.1590/ S0100-40422011000700008

19. E. Hernández-Núñez, H. Tlahuext, R. Moo-Puc, H. Torres-Gómez, R. Reyes-Martínez, R CedilloRivera, C. Nava-Zuazo and G. Navarrete-Vazquez, Synthesis and in vitro trichomonicidal, giardicidal and amebicidal activity of $\mathrm{N}$-acetamide(sulfonamide)-2-methyl-4-nitro- $\mathrm{H}$-imidazoles, Eur. J. Med. Chem. 44 (2009) 2975-2984; https://doi.org/10.1016/j.ejmech.2009.01.005

20. B. R. Brooks, C. L. Brooks, A. D. Mackerell, L. Nilsson, R. J. Petrella, B. Roux, Y. Won, G. Archontis, C. Bartels, S. Boresch, A. Caflisch, L. Caves, Q. Cui, A. R. Dinner, M. Feig, S. Fischer, J. Gao, M. Hodoscek, W. Im, K. Kuczera, T. Lazaridis, J. Ma, V. Ovchinnikov, E. Paci, R. W. Pastor, C. B. Post, J. Z. Pu, M. Schaefer, B. Tidor, R. M. Venable, H. L. Woodcock, X. Wu, W. Yang, D. M. York and M. Karplus, CHARMM: The biomolecular simulation program, J. Comput. Chem. 30 (2009) 1545-1614; https://doi.org/10.1002/jcc.21287

21. P. R. Gerber and K. Müller, MAB, a generally applicable molecular force field for structure modelling in medicinal chemistry, J. Comput. Aided Mol. Des. 9 (1995) 251-268; https://doi.org/10.1007/ bf00124456

22. C. A. Del Carpio, Y. Takahashi and S.-i. Sasaki, A new approach to the automatic identification of candidates for ligand receptor sites in proteins: (I) Search for pocket regions, J. Mol. Graph. 11 (1993) 23-29; https://doi.org/10.1016/0263-7855(93)85003-9

23. A. Miranker and M. Karplus, Functionality maps of binding sites: A multiple copy simultaneous search method, Proteins: Struct. Funct. Genet. 11 (1991) 29-34; https://doi.org/10.1002/prot.34011010

24. S. Thangapandian, S. John, Y. Lee, S. Kim and K. W. Lee, Dynamic structure-based pharmacophore model development: A new and effective addition in the histone deacetylase 8 (HDAC 8 ) inhibitor discovery, Int. J. Mol. Sci. 12 (2011) 9440-9462; https://doi.org/10.3390/ijms12129440

25. A. Wadood, M. Ghufran, S. F. Hassan, H. Khan, S. S. Azam and U. Rashid, In silico identification of promiscuous scaffolds as potential inhibitors of 1-deoxy-D-xylulose 5-phosphate reductoisomerase for treatment of Falciparum malaria, Pharm. Biol. 55 (2017) 19-32; https://doi.org/10.1080/1388 0209.2016.1225778

26. A. M. Clark and P. Labute, 2D depiction of protein-ligand complexes, J. Chem. Inf. Model. 47 (2007) 1933-1944; https://doi.org/10.1021/ci7001473

27. S. Lara-González, P. Estrella, C. Portillo, M. E. Cruces, P. Jiménez-Sandoval, J. Fattori, A. C. Migliorini-Figueira, M. López-Hidalgo, C. Díaz-Quezada, M. López-Castillo, C. H. Trasviña-Arenas, E. Sánchez-Sandoval, A. Gómez-Puyou, J. Ortega-López, R. Arroyo, C. G. Benítez-Cardoza and L. G. Brieba, Substrate-induced dimerization of engineered monomeric variants of triosephosphate isomerase from Trichomonas vaginalis, PLoS ONE 10 (2015) e0141747; https://doi.org/10.1371/journal. pone. 0141747

28. P. Jiménez-Sandoval, J. L. Vique-Sanchez, M. L. Hidalgo, G. Velazquez-Juarez, C. Díaz-Quezada, L. F. Arroyo-Navarro, G. M. Morán, J. Fattori, A. J. Diaz-Salazar, E. Rudiño-Pinera, R. SoteloMundo, A. C. Migliorini-Figueira, S. Lara-Gonzalez, C. G. Benítez-Cardoza and L. G. Brieba, A competent catalytic active site is necessary for substrate induced dimer assembly in triosephosphate isomerase, Biochim. Biophys. Acta - Prot. Proteom. 1865 (2017) 1423-1432; https://doi. org/10.1016/j.bbapap.2017.07.014

29. G. Álvarez, J. Martínez, B. Aguirre-López, N. Cabrera, L. Pérez-Díaz, M. T. de Gómez-Puyou, A. Gómez-Puyou, R. Pérez-Montfort, B. Garat, A. Merlino, M. González and H. Cerecetto, New chemotypes as Trypanosoma cruzi triosephosphate isomerase inhibitors: a deeper insight into the mechanism of inhibition, J. Enzyme Inhib. Med. Chem. 29 (2014) 198-204; https://doi.org/10.3109/147 56366.2013.765415 
30. A. Gómez-Puyou, E. Saavedra-Lira, I. Becker, R. A. Zubillaga, A. Rojo-Dominguez and R. PerezMontfort, Using evolutionary changes to achieve species-specific inhibition of enzyme action studies with triosephosphate isomerase, Chem. Biol. 2 (1995) 847-855; https://doi.org/10.1016/10745521(95)90091-8

31. M. de N. C. Soeiro and S. L. Castro, Screening of potential anti-Trypanosoma cruzi candidates: In vitro and in vivo studies, Open Med. Chem. J. 5 (2011)21-30;https://doi.org/10.2174/1874104501105010021

32. G. Álvarez, B. Aguirre-López, J. Varela, M. Cabrera, A. Merlino, G. V. López, M. L. Lavaggi, W. Porcal, R. Di Maio, M. González, H. Cerecetto, N. Cabrera, R. Pérez-Montfort, M. Tuena de GómezPuyou and A. Gómez-Puyou, Massive screening yields novel and selective Trypanosoma cruzi triosephosphate isomerase dimer-interface-irreversible inhibitors with anti-trypanosomal activity, Eur. J. Med. Chem. 45 (2010) 5767-5772; https://doi.org/10.1016/j.ejmech.2010.09.034

33. C. G. Benítez-Cardoza, D. A. Fernández-Velasco and J. L. Vique-Sánchez, Triosephosphate isomerase inhibitors as potential drugs against Clostridium perfringens, Chem. Sel. 5 (2020) 2365-2370; https://doi.org/10.1002/slct.201904632

34. J. L. Vique-Sánchez, L. A. Caro-Gómez, L. G. Brieba and C. G. Benítez-Cardoza, Developing a new drug against trichomoniasis, new inhibitory compounds of the protein triosephosphate isomerase, Parasitol. Int. 76 (2020) Article ID 102086; https://doi.org/10.1016/j.parint.2020.102086

35. A. Téllez-Valencia, S. Avila-Ríos, R. Pérez-Montfort, A. Rodríguez-Romero, M. Tuena de GómezPuyou, F. López-Calahorra and A. Gómez-Puyou, Highly specific inactivation of triosephosphate isomerase from Trypanosoma cruzi, Biochem. Biophys. Res. Commun. 295 (2002) 958-963; https://doi. org/10.1016/s0006-291x(02)00796-9

36. B. Hernández-Ochoa, G. Navarrete-Vázquez, C. Nava-Zuazo, A. Castillo-Villanueva, S. T. Méndez, A. Torres-Arroyo, S. Gómez-Manzo, J. Marcial-Quino, M. Ponce-Macotela, Y. RufinoGonzález, M. Martínez-Gordillo, G. Palencia-Hernández, N. Esturau-Escofet, E. Calderon-Jaimes, J. Oria-Hernández and H. Reyes-Vivas, Novel giardicidal compounds bearing proton pump inhibitor scaffold proceeding through triosephosphate isomerase inactivation, Sci. Rep. 7 (2017) Article ID 7810; https://doi.org/10.1038/s41598-017-07612-y 